Weber, Jacqueline; Laqua, Daniel; Williamson, Adam; Husar, Peter; Schober, Andreas:

\title{
Capacitive sensor concept for monitoring neuronal activity in vitro
}

Zuerst erschienen in: $\quad$ Biomedical Engineering = Biomedizinische Technik. - Berlin [u.a.] : de Gruyter. - 57 (2012), Suppl. 1, Track-G, p. 1055-1058.

Erstveröffentlichung: 2012-08-31

ISSN (online): $\quad$ 1862-278X

ISSN (print): 0013-5585

DOI: $\quad 10.1515 / \mathrm{bmt}-2012-4074$

[Zuletzt gesehen: 2019-08-13]

„Im Rahmen der hochschulweiten Open-Access-Strategie für die Zweitveröffentlichung identifiziert durch die Universitätsbibliothek IImenau. “

"Within the academic Open Access Strategy identified for deposition by Ilmenau University Library."

„Dieser Beitrag ist mit Zustimmung des Rechteinhabers aufgrund einer (DFGgeförderten) Allianz- bzw. Nationallizenz frei zugänglich."

"This publication is with permission of the rights owner freely accessible due to an Alliance licence and a national licence (funded by the DFG, German

Research Foundation) respectively." 


\title{
Capacitive Sensor Concept for Monitoring Neuronal Activity in Vitro
}

\author{
J. Weber ${ }^{1}$, D. Laqua ${ }^{1}$, A. Williamson ${ }^{2}$, P. Husar ${ }^{1}$ and A. Schober ${ }^{2}$ \\ ${ }^{1}$ Department Biosignal Processing (BSV), TU Ilmenau, Ilmenau, Germany \\ 2 Department Nanobiosystemtechnique (NBST), TU Ilmenau, Ilmenau, Germany
}

\begin{abstract}
-
The goal of this work is to monitor the activity of neurons in order to better understand information distribution within neural networks. Unfortunately galvanic contact with electrodes can disturb cell growth. Therefore in vitro measurements without cell contact are preferred. Capacitive sensors offer a flexible and noncontact way of implementing a sensor for monitoring neuronal activity. The capacitance of the sensor output is numerically calculated using the simulation tool ANSYS Maxwell simulation. This model was used to simulate the behaviour of the sensor depending on one axon, the location of the action potential and the distance between sensor and axon. Efficient and selective electrical recording of neuronal activity requires multielectrode arrays at the micrometer scale. First test measurements were performed on an axon model with a scaled dimension of $300: 1$ raising the dimensions of the system into the millimeter range. The first element of the amplifier system is an ultra-low bias current operational amplifier (input current of $100 f A$ max.) One implementation was investigated without any bias current path. For avoiding a polarisation of the sensors a hardware bias current path was implemented at the first stage in two other implementations. One with a resistance of $100 \mathrm{k} \Omega$ and the other with two diodes in opposite directions. The simulation shows a typical potential distribution in the sensor decreasing with rising distance between sensor and axon as predicted. First test measurement with a sine-wave as source signal have provided results with a SNR for further signal processing. A measuring system for capacitive monitoring of neuronal cells tested on a dimension of $300: 1$ is presented. Further work will show measurements on an unscaled system with neuronal cell structures grown around a multielectrode array.
\end{abstract}

Keywords - neuronal activity, capacitive sensor, multielectrode arrays

\section{INTRODUCTION}

The growth and in vitro study of neuronal cell cultures remain forefronts in medical research, however the study of neuronal cells in vivo in the human body remains a more complex problem due to significantly more complicated methods of investigation. Such complicated methods are nec- essary but have lead to less understanding of the functioning and interaction of neuronal cells in vivo. This is unfortunate because, with better understanding, cultured cells could be more effectively used to replace dead or failing cells in the human body, possibly healing or reversing some handicaps. The main problem is that researchers are not sure how much data collected from in vitro study is actually comparable with in vivo behaviour, leading to problems, for example, in the definition of cell communication. Cells certainly fire, but do they communicate? The preferred solution would be the creation of a biocompatible, contactless measurement system for the detection of cell communication to completely minimize any influence by the measurement method itself on cell behaviour. This would bring data collected in vivo one step closer to accurately representing behaviour in vivo.

The work presented here estimates the dimensions required for contactless measurements of the activity of cultured neuronal cells measured by capacitive sensors and finds a possible way to validate the measurement system should it be developed. Important to note is that the detection of the activity and not the absolute amplitude of the signal is important.

\section{MATERIAL AND METHODS}

Before designing a circuit the expected measurement values needed to be estimated with finite element method (FEM) calculations. Channels spanning the axon of an individual neuron allow the exchange of potassium and sodium ions between membranes which produce the so-called action potential, the cell firing. However displaying this neuronal activity on a FEM grid is not possible, thus a suitable model was designed with a static charge representing the action potential of a single axon to be measured by a single sensor, shown in Figure 1.

As seen, the axon was built with a chain of cylinders representing regions of the axon surrounded by a Myelin sheath and with adjacent cylinders separated by membranes (nodes of Ranvier). The potential across the membrane in a rest state before or after ion flux is called the resting membrane potential (rmp). When depolarization occurs a neuron fires an action potential across the membrane with a magnitude of 


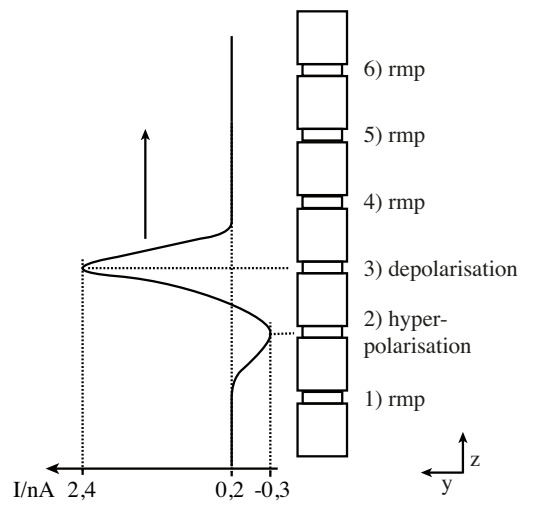

Fig. 1: The principle of the static simulation, position 2

$55 \mathrm{mV}$. When channels remain open this action potential can reach $70 \mathrm{mV}$ and is called hyperpolarization. In the simulation the static charges are placed at the nodes of Ranvier and the magnitude of the charges are changed for each of the three situations yielding measured membrane currents of $0.2 n A$ for the resting membrane potential, $2.4 n A$ for depolarization and $0,3 n A$ for hyperpolarization [1]. Transmission of the action potential was realized by alternating the location of the various static charges between membrane positions. Additionally an analysis on the effect of the distance between the axon and the sensor was performed in the y-direction with steps of $0.1 \mu \mathrm{m}$ and in the z-direction with steps of $20 \mu \mathrm{m}$.

Figure 2 shows the simulated excitation principle.

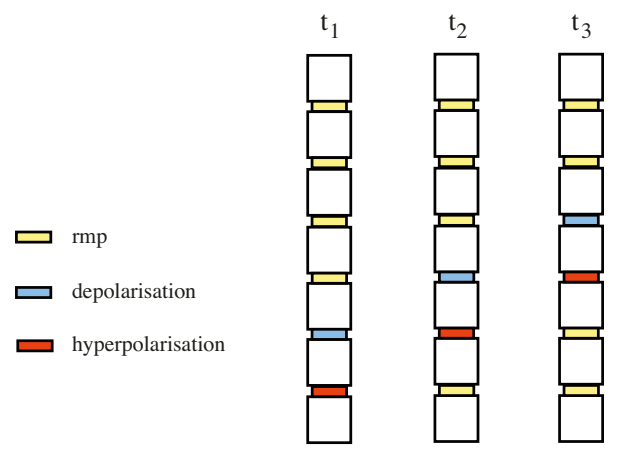

Fig. 2: Simulated excitation principle

Many standard capacitive sensor concepts existed before the starting this work, however here capacitors are created without invested voltage. The action potential when placed in the area of the passivated electrode surface produces a measurable capacitance across the passivation. With the electrode at $0 V$, the cells invest the voltage. The implemented dimensions are shown in table 1 .

\begin{tabular}{lll} 
area & diameter $[\mathrm{mm}]$ & hight $[\mathrm{mm}]$ \\
\hline sensor & 0,05 & 0,001 \\
node of Ranvier & 0,008 & 0,005 \\
myelin sheath & 0,01 & 0,02 \\
ground plane & 4 & 0,2 \\
plexiglass & 4 & 1 \\
glass plane & $0,1 \times 0,8$ & 2,1 \\
culture medium & 5 & 5 \\
\hline
\end{tabular}

Table 1: Implemented dimensions

For practical implementation the operational amplifier opa129 was selected for several of its advantageous characteristics. Figure 3 shows the basic principle of the measurement system with an amplification factor of 100 . The capacitors are used for stabilizing the supply voltage and $\mathrm{R}$ represents the bias resistor. Different variants of the bias resistor were tested $(20 \mathrm{GW}, 1 \mathrm{MW}, 475 \mathrm{~kW}, 100 \mathrm{~kW}$ and $10 \mathrm{~kW})$. $100 \mathrm{~kW}$ resistors were found to be most appropriate. Because a higher amplification was required a second amplifier stage was introduced. An additional shielding around the sensor was used for reducing the noise of the signal.

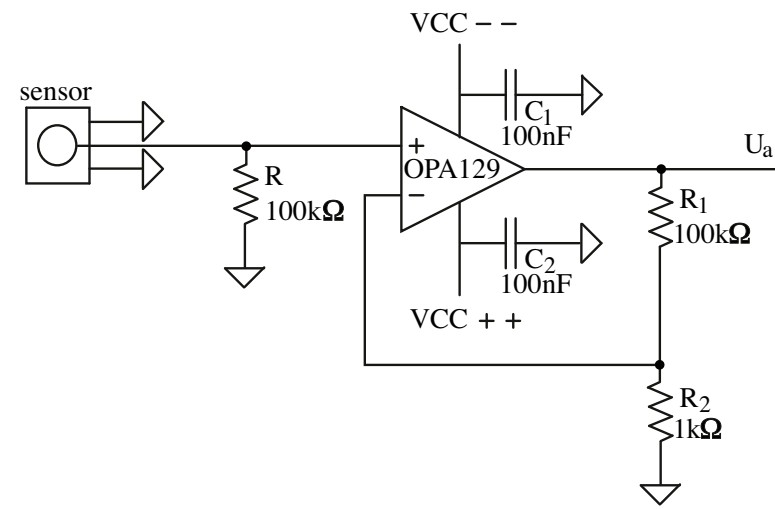

Fig. 3: Basic structure of the measuring system

In order to validate the measuring system a suitable signal source had to be found. The model used in the simulation was also applied in the practical realization.

The physical dimensions of the real system and the sensor surface were magnified by a factor of 300 compared to the dimensions of the simulation. The nodes of Ranvier were realized with steel pins isolated from one other. The charge used to represent the action potential was generated at a frequency of $480 \mathrm{~Hz}$ which for neuronal signal transmission is realistic and is slow enough to be comparable to the static charge used 
in the FEM simulation. Charges placed in the nodes of Ranvier realized membrane currents of $0.2 \mathrm{~mA}$ for the rmp, $2.4 \mathrm{~mA}$ for depolarization and $0.3 m A$ for hyperpolarization. Figure 4 shows the voltage-controlled current source which was used for sinusoidal signal generation. All power sources have been supplied through a voltage source and the negative hyperpolarization was provided with an inverter ahead of the circuit.

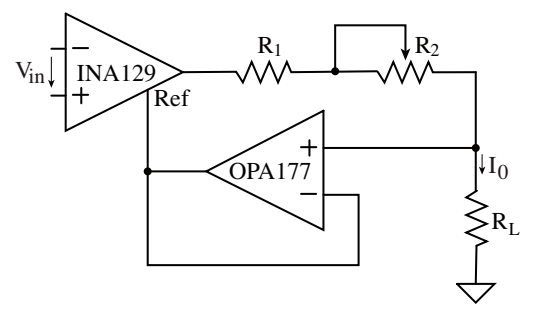

Fig. 4: Voltage-controlled current source

The measurement results were recorded with software using a sampling rate of $10 \mathrm{kHz}$. As in the simulation, the experimental transmission of the action potential was realized by alternating the location of the charge between membrane positions. An analysis on the effect of the distance between the axon and the sensor was again performed in the y-direction but with steps of $0.3 \mathrm{~mm}$ and in the $\mathrm{z}$-direction and with steps of $60 \mathrm{~mm}$ due to the magnification in scale. As the recorded data was relatively noisy some post-measurement filtering was done by the addition of a Butterworth bandpass filter at $480 \mathrm{~Hz}$. Further processing of the data included analyzing peak-to-peak values per measurement period with an average of the peak-to-peak values showing the measured value for each excitation position.

\section{RESULTS}

\begin{tabular}{llllll} 
& $t_{1}[\mathrm{pV}]$ & $t_{2}[\mathrm{pV}]$ & $t_{3}[\mathrm{pV}]$ & $t_{4}[\mathrm{pV}]$ & $t_{5}[\mathrm{pV}]$ \\
\hline$Y_{0}$ & $-2,39$ & $-5,02$ & $-2,52$ & $-0,96$ & $-0,67$ \\
\hline$Y_{1}$ & $-1,60$ & $-4,79$ & $-1,80$ & $-0,42$ & $-0,39$ \\
\hline$Y_{2}$ & $-1,26$ & $-3,22$ & $-1,46$ & $-0,35$ & $-0,09$ \\
\hline$Y_{3}$ & $-0,87$ & $-2,21$ & $-1,09$ & $-0,17$ & $-0,03$ \\
\hline$Y_{4}$ & $-0,41$ & $-0,89$ & $-0,65$ & $-0,12$ & $-0,02$ \\
\hline$Y_{5}$ & $-0,08$ & $-0,51$ & $-0,38$ & $-0,07$ & $-0,002$ \\
\hline \hline$Z_{0}$ & $-2,38$ & $-4,84$ & $-2,46$ & $-1,19$ & $-0,65$ \\
\hline$Z_{1}$ & $-1,05$ & $-2,51$ & $-1,20$ & $-0,47$ & $-0,37$ \\
\hline$Z_{2}$ & $-0,42$ & $-1,59$ & $-0,56$ & $-0,16$ & $-0,02$ \\
\hline
\end{tabular}

Table 2: Simulation results
To simplify the description of the results $Y_{0}$ stands for the position of the axon being measured by the sensor in the simulation at the distance $Y_{1}=0.1 \mu \mathrm{m}$ or $Y_{2}=20 \mu \mathrm{m}$, and in the experiment at the distance $Y_{1}=0.3 \mathrm{~mm}$ or $Y_{2}=0.6 \mathrm{~mm}$. $Z_{0}$ corresponds to the position $Y_{0}$ rotated $90^{\circ}$ with respect to the $\mathrm{x}$-axis in the simulation at the distance $Z_{1}=20 \mu \mathrm{m}$ or $Z_{2}=$ $-20 \mu \mathrm{m}$, and in the experiment $Z_{1}=6 \mathrm{~mm}$ or $Z_{2}$ at $-6 \mathrm{~mm}$. The values at 5 times are taken to describe the excitation supply positions in the node of Ranvier. For a better comparison of the results all values were normalized to the maximum value of a distance position.

The resulting negative potential in each simulation is visible in table 2. Consideration of the only the magnitude is adequate as only the state change should be measured and exact sizes and signs are less relevant at this time. The potential is generated in the $\mathrm{pV}$ region which is at a signal source intensity of $\mathrm{nA}$ and is absolutely realistic. Clearly visible are the maximum values at excitation $t_{2}$ which decrease with the distance between the sensor and axon. In the $t_{1}$ and $t_{2}$ excitation the effect with increasing distance is also visible. At excitations $t_{4}$ and $t_{5}$ the changes are relatively minimal. This is explained by the fact that the distance to the sensor is already relatively large and the effect of changing distance is minimal. These results were expected as the excitation $t_{2}$ can be found right in the middle of the sensor surface. The space between $t_{2}$ and $t_{1} / t_{3}$ in the simulation was at position $Y_{0}$ from $5 p V$ to $2.5 p V$ and drops down to a minimal $0 p V$.

The dependence of the axon position in z-direction is also visible and the maximum is at excitation $t_{2}$. Interesting to note here is the significantly greater attenuation of the potentials when the axon is moved away from the ground plane. With the $Z_{1}$ axon position the $t_{2}$ value is roughly halved while minimizing the value at the opposite position $Z_{2}$ by a factor of three. In practical measurement a maximum value achieved at excitation $t_{2}$ is also recorded.

Measurements in air do not show a special constant change in the values of the distance change. The values expected by the simulation are accredited but not fundamentally the same as the simulation values. The gradation between $t_{2}$ and the surrounding excitations is not so clearly developed. Visible is a relatively large range of values between distance $Y_{1}$ and $Y_{2}$ which suggests that a distance of $0.6 \mathrm{~mm}$ has a high influence on sensor potential. Measurements in z-direction are at a relatively large distance between the $t_{2}$ and surrounding excitation positions.

The space between $t_{2}$ and $t_{1} / t_{3}$ in the real measurements in air was at position $Y_{0}$ from $47 n V$ to $39 n V$ and drops down to a minimal $25 n V$. Measurements in cultured mediums show a significant improvement in signal intensity about a factor of three compared to measurements in air. Also the relatively 
large range of values which was visible in the air measurement between distance $Y_{1}$ and $Y_{2}$ is shown in measurement in culture medium between distance $Y_{2}$ and $Y_{3}$. This confirms the amplification of the signal transmission through the cultured medium. From the $Y_{3}$-distance a slight increase in the $t_{2}$-excitation is still present but not significant enough to detect a stimulation. The space between $t_{2}$ and $t_{1} / t_{3}$ in the real measurements in cultured medium was at position $Y_{0}$ from $173 n V$ to $160 n V$ and drops down to a minimal $145 n V$.

\section{DISCUSSION}

Several limitations which may introduce error on the numerical and experimental work are apparent. Ion concentrations inside and outside the axon which create the action potential were not calculated in the simulation and axons themselves do not have perfectly cylindrical surfaces Additionally, in the simulation and experiment the distances between the nodes of Ranvier were held constant but in reality such distances can vary. Simulation values assumed ideal conditions (for example a noise free environment) which are not possible to achieve in experiments. Another possible source of error is the direction of current. In the cell the ion current flows from extracellular to intracellular or in the reverse direction, but in the simulation the current direction was simply rotated $90^{\circ}$.

The measured values show a standard deviation of 0.26 in air and of 2.04 in culture mediums. This deviation can be explained by the properties of the filter. The magnitude of the current inside an actual cell is less than the experimentally realized current by a factor of $10^{6}$, and that subsequently increases the gain needed to detect the signal by the same factor. The measured potential at the sensor reaches $25 \mathrm{mV}$ and falls back not quite to zero. This is due to several factors. The influence of the modifications is still visible, even if only minimally and the noise component is not filtered out completely. The biggest challenge for future work is the frequency modulation of the signal transmission, because the filtering process of the signal will be more complex.

An improvement of the measured values in the cultured medium by a factor of three is clearly visible and can be attributed to the enhanced conductivity of the medium compared to air. The measurements in the cultured medium correspond to the simulation much more than measurements in air. Important is also the fact that the cultured medium resides in a container and the possibility cannot be excluded that the edge surfaces of the cultured medium change the field features and the subsequent measured signal. At this time it is not absolutely certain that increasing the dimensions and increasing the current would have an effect on the generated fields.
In general the differences between simulations and measurements can be explained by several factors. Firstly the relative permittivity of air in the FEM is taken to be a constant real number and is in experiment only slightly different however the relative permittivity of the metal components could likely differ more significantly between reality and simulation. Secondly the non-ideal dimensions caused by the use of real steel pins for the nodes of Ranvier with slightly varying distances between the pins. Interesting to note is that the less than exact dimensions, spacing, and placement of the pins would be closer to the real conditions of the cell. Thirdly, and likely the largest source of error, is the use of metal for the nodes of Ranvier. Lastly, in the simulation only one sensor was considered and the complete circuitry was neglected and obviously the type of circuitry plays a in the measured field characteristics.

\section{CONCLUSION}

The present work describes the creation of a measuring system for the contactless, capacitive detection of activity in cultured neuronal cells with the aim of improving the comparability of in vitro data to in vivo behaviour. Simulations showed that the measured signals are in the expected range of $\mathrm{pV}$ with the action potential showing the measured signal increased by a factor of five compared to the rmp.

The measuring system contained two amplifier stages realized by non-inverting amplifiers and allowed a amplification of $10^{4}$. In the verification of the bias resistor a resistance of $100 \mathrm{k} \Omega$ showed to be most suitable. To validate the simulations of the measuring system an experimental replica was constructed. First measurements in air and further measurements in nutrient solutions confirmed the functionality of the measurement system but highlighted several weak points for improvement, for example the amplifier offset which is required for raising the signal and frequency modulation. The experimental measurements showed a signal increase during depolarization by a factor of 1.3 compared to the rmp state. This factor represents an expected value for the measurement of firing cells.

\section{REFERENCES}

1. Toshiaki Nakajima Hisanori Hazama Masao Omata. Effects of pirmenol on action potentials and membrane currents in single atrial myocytes. Second Department of Internal Medicine, Faculty of Medicine, University of Tokyo, 7-3-1 Hongo, Bunkyo-ku, Tokyo 113, Japan 1997. 